\title{
Experiencia de 10 años con el manejo de la colpocistouretropexia suprapúbica
}

\author{
Antonio Lomanto Morán*; Jacinto Sánchez Angarita**; Pedro Jiménez M.; Lucio Guerra G.; Pedro Rozo R.***
}

RESUMEN: Se analizaron 500 casos de I.U.E. en la mujer, mediante la Colpocistouretropexia suprapúbica, entre el 1 de julio de 1977 y el 30 de junio de 1986, en los Servicios de Ginecología y Urología del Instituto Materno Infantil y el Centro Hospitalario San Juan de Dios, y de Ginecología de la Clínica Fray Bartolomé de las Casas de Bogotá.

La frecuencia de la Colpocistouretropexia fue de $3.8 \%$. El $56.2 \%$ presentaba IUE de Grado II y el $\mathbf{4 3 . 8} \%$ Grado III. El $96 \%$ tenía celes concomitantes discriminados así: Grado I 34.8\%, Grado II $54.4 \%$ y Grado III $6.8 \%$. El $12.4 \%$ tenía antecedente de cirugía de Kelly y el $1 \%$ de Colpocistouretropexia suprapúbica.

Se practicaron los siguientes exámenes paraclínicos:

Urocultivo: 100\%, Citología vaginal: 93.7\%, Citoscopia: 100\%, Urografía: 40\%, Urodinamia: 11.2\%, Cistometría: $19.6 \%$, Cistografía: $14 \%$.

Se practicaron los siguientes procedimientos quirúrgicos:

Suprapúbicos: $35.6 \%$, Suprapúbico + Colporrafia Posterior: $\mathbf{4 9 . 2} \%$.

Suprapúbico + Histerectomía Abdominal: 12\% - Suprapúbico + Pomeroy $1.8 \%$.

Suprapúbico + Cirugía Vaginal 1.6\%.

El tiempo quirúrgico fue de 1 hora 45 minutos. En el 54\% se utilizó la incisión de Pfannestiel. En el 71.8\% se utilizó material no absorbible. En el $\mathbf{8 7 . 1 4 \%}$ se utilizó drenes. En el $\mathbf{5 1 . 8 \%}$ las intervenciones fueron realizadas por Residentes.

Las complicaciones intra-operatorias fueron:

Ruptura vesical $1.6 \%$-Hematuria $8.4 \%$-Hemorragia $5.2 \%$.

Las complicaciones post-operatorias fueron:

Infección urinaria 16.8\%-Infección de la herida $9 \%$ - Retención urinaria $16.8 \%$-Dolor suprapúbico $11.14 \%$.

El promedio de permanencia de la sonda dude $4.8 \%$. La estancia hospitalaria fue de $32.8 \%$, a dos años $24.8 \%$ y de 3 a 5 años $31 \%$. La eficacia fue del $96.4 \%$.

Con base en la experiencia durante 10 años en nuestros servicios, consideramos que la Colpocistouretropexia suprapúbica es un procedimiento eficiente en el manejo de la IUE en la mujer sin desconocer la morbilidad que conlleva.

\section{PALABRAS CLAVES: Colpocistouretropexia suprapúbica}

SUMMARY: 500 hundred cases of I.U.E., in woman have been analized by means of retropubic colposuspension, between july last, 1977 and june 30, 1986 in the Gynecological and Urology services of the Materno-Infantil Institute, San Juan de Dios Hospital and of the Gynecology of the Fray Bartolomé de las Casas Clinic in Bogotá.

The frecuency of the retropubic colposuspension was of $3.8 \%$. The $56.2 \%$ presented I.U.E. Grade II and the $43.8 \%$ Grade III. The $96 \%$ have concomitant prolapse discriminated as follows: Grade I: $34.8 \%$; Grade II: $54.4 \%$ and Grade III: $6.8 \%$. The $12.4 \%$ had previous surgery of Kelly and the $1 \%$ of Retropubic Colposuspension.

The following paraclinic surveys were performed: hy: $14 \%$

Uroculture: 100\%; Vaginal cytology: 93.7\%; Cystoscopy: 100\%; Urography: 40\%; "Urodynamies": 11.2\%; "Cystometry" 19.6\%; Cystograp-

The following surgery Procedures were performed:

Suprapubics: $35.6 \%$; suprapubics + later colporrafy: $49.2 \%$.

Suprapubics + abdominal hysterectomy: 12\%- Suprapubics + Pomeroy: $1.8 \%$.

Suprapubic + Vaginal surgery $1.6 \%$.

The surgery time was of 1 hour 45 minutes. In $\mathbf{5 4 \%}$ were utilized de Pfnnestiel incision - In $\mathbf{7 1 . 8 \%}$ were utilized non-absorbable material. In $87.14 \%$ were used drains? (drenes). In $51.8 \%$ the surgery was performed by Residents.

The intra-surgeries complications were:

Vesical rupture $1.6 \%$ - Hematury $8.4 \%$, hemorrhage: $5.2 \%$.

Post-surgery complications were:

Urinary infection: $16.8 \%$ - wound infection: $9 \%$; urinary retention: $16 \%$ - suprapubic pain: $11.14 \%$.

The average of the permanence of the catheter was of $4.8 \%$. The permanence on the hospital was of $32.8 \%-$ two years $24.8 \%$ and 3 to 5 years $31 \%$. The efficacy was of $96.4 \%$.

Based on the experiency during 10 years in our services, we consider that the Retropubic Colposuspension is an efficient procedure in the managing of the I.U.E. in woman, recognizing the concerning morbility.

KEY WORDS: Retropubic colpopexy.

Profesor Asociado - Departamento de Ginecología y Obstetricia-Facultad de Medicina Universidad Nacional de Colombia.

** Profesor Asistente-Departamento de Ginecología y Obstetricia-Facultad de Medicina-Universidad Nacional de Colombia.

*** Residentes III-Departamento de Ginecología y Obstetricia Facultad de Medicina, Universidad Nacional de Colombia.

\section{Introducción}

1. La incontinencia urinaria es un problema permanente para muchas mujeres y la necesidad de su valoración y tratamiento depende de la frecuencia y magnitud del problema. En otras mujeres, la pérdida urinaria es mínima, y ocurre 
ocasionalmente y en tales condiciones no se considera problema. Está indicada la corrección de este trastorno cuando crea una auténtica invalidez desde el punto de vista social y la paciente solicita valoración adicional y una solución al problema. Los adelantos registrados en los últimos 30 años han propiciado un mejor conocimiento de la incontinencia urinaria en la mujer (1).

2. La incontinencia de esfuerzo genuina tiene lugar cuando la presión intravesical a consecuencia de un aumento de presión intraabdominal excede de la resistencia producida por los mecanismos de cierre uretrales, en ausencia de actividad de la vejiga. Otras formas de incontinencia pueden confundirse con la incontinencia urinaria de esfuerzo pura por la similitud de síntomas y la capacidad de producir el signo clínico de pérdida de orina sin maniobras de esfuerzo. Los transtornos asociados con contracción del detrusor (inestabilidad del detrusor), aumento de presión intravesical (poca adaptabilidad) o incremento de la orina residual (incontinencia por rebozamiento) pueden tener asociados síntomas "producidos por el stress". La inestabilidad uretral depende de la relajación refleja de la uretra sin contracción del detrusor (2-3).

\section{La incontinencia de esfuerzo genuina puede depender de:}

1. Un mecanismo de cierre uretral intrínsecamente incompetente (4).

2. Un mecanismo de continencia desplazo y deformado desde el punto de vista anatómico, pero intrínsecamente intacto; o

3. Una combinación de ambos (2)

Los síntomas incontinencia de esfuerzo y urgencia, se han identificado como importantes para diferenciar entre la incontinencia de esfuerzo genuina y o vejiga inestable, y desde hace largo tiempo está comprobado que las pacientes con urgencia e incontinencia de urgencia responden en medida escasa a la cirugía acostumbrada. El análisis crítico de estos síndromes por estudios urodinámicos han ayudado a precisar el valor y las limitaciones del interrogatorio en la asistencia de la incontinencia.

El signo de la incontinencia de esfuerzo genuina es la observación directa de la pérdida de orina procedente de la uretra externa inmediatamente después de un aumento de la presión intraabdominal. Si la pérdida se produce unos pocos segundos después del aumento de la presión intraabdominal probablemente no esté relacionado con la incontinencia urinaria de esfuerzo genuina sino que está más a menudo asociada con una actividad inapropiada del músculo detrusor provocada por el estímulo (5).

La disponibilidad de investigaciones urodinámicas de ninguna manera debe sustituir al interrogatorio y la exploración física acostumbrada, que siguen siendo el aspecto central del estudio de la paciente con incontinencia urinaria de esfuerzo, pero ha permitido revalorar la importancia de los datos que se obtienen de esta manera. La valoración urodinámica ha revelado que hay algunos grupos de síntomas que permiten predecir con bastante seguridad el estado del detrusor $\mathrm{y}$ otros que no son útiles.

\section{Diagnóstico}

Se han introducido un importante número de métodos para evaluar las pacientes con incontinencia urinaria de es- fuerzo (3): El urocultivo, la urografía, la cistouretrograma estático, la uretrocistoscopia (13-15).

La urodinamia: es útil dentro del estudio de pacientes con incontinencia. Tiene indicación principal cuando hay síntomas que sugieren la posibilidad de inestabilidad, lo mismo que en las pacientes que ya han sido sometidas a cirugía para incontinencia, habiendo ésta fracasado, lo mismo que las que tienen algún trastorno neurológico o incontinencia (16-18).

\section{Tratamiento}

La comprensión de las bases del buen éxito en la cirugía para la incontinencia urinaria de esfuerzo se debe mucho a los estudios de Enhorning y col. (Enhorning, 1961; Enhorning, Miller y Hinman, 1964) y de Hodgkinson (1960). Enhorning comprobó que la continencia se logra cuando la unión uretrovesical y la porción proximal de la uretra se elevan hacia la zona abdominal de presión. La presión intrauteral en estado normal es mayor que la presión vesical; cualquier aumento de la presión abdominal se transmitirá igualmente a la vejiga y la porción proximal de la uretra, lo cual conservará el gradiente de presión entre vejiga y uretra y asegurará presión positiva de cierre en el cuello vesical y uretra (20).

Cuando ocurre insuficiencia verdadera de esfuerzo, se advierte uno o más de los siguientes datos patológicos:

1. Cambio de la posición del cuello vesical, de manera que cualquier aumento de la presión intraabdominal produce aumento semejante en la presión del detrusor intravesical, pero no de la presión intrauretral. La presión del detrusor excederá de la presión intrauretral y ocurrirá incontinencia. Ello puede acompañarse de cistocele, o no.

2. Longitud funcional escasa en la uretra.

3. El cuello vesical adopta disposición infundibuliforme por el ejercicio o al pujar (lo cual se acompaña de contracción del destrusor (21).

Las finalidades de la cirugía son:

1. Elevar el cuello de la vejiga y la uretra proximal para que recuperen la posición anatómica original, dentro de la zona de presión abdominal.

2. Impedir que el cuello vesical adopte disposición infundibuliforme al aumentar la cantidad de tejido de sostén alrededor de esta área.

3. Aumentar la resistencia uretral (22).

Selección de la técnica quirúrgica. El lugar de la reparación anterior para el tratamiento de la incontinencia por esfuerzo es objeto de discusión. Donald (1988) fue uno de los primeros en describir esta intervención, mientras que Kelly (1913) se recuerda por las suturas que llevan su nombre colocadas en el cuello de la vejiga. Como entonces, actualmente la repación anterior la utilizan muchos ginecólogos para corregir prolapso de pared vaginal e incontinencia urinaria de esfuerzo. Estudios en la literatura indican que 48 cie 90\% (Low, 1967, Greem 1975) curan subjetivamente, pero la valoración objetiva disminuye la proporción de curaciones hasta cifras tan bajas como de 38\% (Stanton y Cardozo, 1979; Gómez y Lomanto, 1983, 48\%). Sin embargo es indudable que hay médicos que llevan a cabo selección cuidadosa de pacientes y son técnicamente capaces de producir una cifra muy elevada de curaciones de la inconti- 
nencia de esfuerzo, pero la proporción con buen éxito global parece ser más baja que para las operaciones de suspensión suprapúbica del cuello vesical $(28,29)$.

A partir de 1949 se comenzaron a describir una serie de técnicas quirúrgicas para la corrección de la incontinencia urinaria de esfuerzo, cuyo objetivo fundamental es la elevación del cuello vesical o tejidos parauretrales a estructuras posteriores del pubis, de la índole del cartílago de la sínfisis del pubis, del periostio de la rama del pubis, vaina de los rectos o a los ligamentos de Cooper entre las cuales están las técnicas de MARSHALL, MARCHETTI Y KRANTZ en 1949, PEREYRA en 1959, BURCH en 1961, TANAGHO en 1976, STAMEY en 1980, que han dado una efectividad del 90 a $93 \%$, en pacientes bien seleccionadas (26, 30-34).

La operación de Marshall, Marchetti, Krantz, propuesta en 1949, tiene una proporción global de buenos resultados de 90 a 93\% (Marshall y Segaul, 1968). La técnica descrita, originalmente incluye material de sutura crómico del número 1 puesto junto a la uretra y luego atravesando el periostio de la sínfisis del pubis. Los problemas relacionados con la operación incluyen osteitis del pubis 5\%, retención urinaria prolongada, enterocele e inestabilidad persistente del detrusor.

Para evitar los efectos de la intervención sobre la propia uretra, disminuir la osteitis del pubis, y porque los cirujanos han reconocido que el sostén de la uretra podía lograrse elevando la fascia vesico-vaginal a los lados de la uretra, Burch en 1961 introdujo puntos entre ella paravaginal y el ligamento de Cooper homolateral. Este último invariablemente es resistente y bien definido. Al atar los puntos, el cuello de la vejiga y la pared vaginal anterior se elevan y mejoraba el dominio de la incontinencia.

Complicaciones de la uretrocolpopexia suprapúbica

Pueden dividirse en operatorias y postoperatorias: (35)

Entre las transoperatorias tenemos:

1. Hemorragia de las venas perivesicales.

2. Lesiones vesicales y uretrales.

3. Desgarro del fondo de saco vaginal.

Entre las postoperatorias tenemos:

1. Retención urinaria.

2. Infección urinaria.

3. Micción urgente y polaquiuria.

4. Hematoma retropúbico.

5. Enterocele.

6. Dispareunia.

7. Recurrencia de la incontinencia.

8. Inestabilidad vesical.

La técnica de Burch, fue modificada por Tahagho en 1976: muchos procedimientos envuelven disección extensa de la unión uretrovesical y de la uretra proximal con una gran predisposición a la fibrosis, cicatrización y denervación de estos órganos normales. El procedimiento de Burch modificado (técnica de Tanagho) (32) incluye:

1. Mínima disección dentro de $2 \mathrm{cms}$ de la unión uretrovesical y de la uretra.

2. Colocación de las suturas a través de la fascia paravaginal en lugar del tejido parauretral.

3. Uso de dos suturas de material no absorbible en cada lado, uno en la unión uretrovesical y otro a nivel de la uretra media.
4. Remoción del tejido graso lateral a las suturas estimulando la fibrosis y la fijación al retropubis.

5. Facilitando la colocación de suturas al ligamento de Cooper.

Aunque Beck and McCormic reportaron excelente rata de cura por vía vaginal para el tratamiento de la incontinencia urinaria de esfuerzo, Stanton y otros reportaron menos ratas de curas con el reparo vaginal anterior cuando se compara con la colpocistouretropexia. Durante un año de seguimiento postoperatorio, Mundy reportó una rata de cura subjetiva del $89 \%$ versus una rata de cura objetiva del $73 \%$ después de un procedimiento de colposuspensión (36). En el mismo estudio, el procedimiento de Stamey resultó con una eficacia del $76 \%$ subjetiva en posición de una cura del $40 \%$ objetivamente. Similarmente Weil and asociados reportaron una rata de cura objetiva del $91 \%$ después de uretropexia de Burch en oposición de una rata de cura objetiva del $50 \%$ después del procedimiento de Pereyra y del $57 \%$ después del reparo vaginal anterior (37).

\section{Objetivos}

En el Departamento de Ginecología y Obstetricia de la Facultad de Medicina de la Universidad Nacional, desde 1977 se viene realizando la uretrocolpopexia suprapúbica según la técnica de Burch y Tanagho para el manejo de la incontinencia urinaria de esfuerzo de la mujer (38) y el propósito de nuestra investigación es evaluar la eficacia de la Uretrocolpexia Suprapúbica, en 500 pacientes de la Sección de Urología del Hospital San Juan de Dios, de la Clínica de Incontinencia del Instituto Materno Infantil y del Servicio de Ginecología de la Clínica Hospital Fray Bartolomé de las Casas de Bogotá, durante un período de 10 años comprendidos entre el 1 de enero de 1978 y el 31 de diciembre de 1987 y cuyos objetivos específicos fueron los siguientes:

1. Identificación de las pacientes.

2. Evaluación de las pruebas diagnósticas.

3. Valoración de la eficacia de la técnica quirúrgica

4. Identificación de las complicaciones intra y postoperatorias.

5. Manejo de la sonda vesical y de la estancia hospitalaria.

6. Seguimiento a 3, 6 y 12 meses, 1,2 y 5 años.

7. Valoración de la persistencia y recidiva de la incontinencia urinaria de esfuerzo.

\section{Material y métodos}

Este trabajo es un estudio retrospectivo de tipo descriptivo realizado en el Departamento de Ginecología y Obstetricia de la Facultad de Medicina de la Universidad Nacional en donde se evalúa la eficacia de la Uretrocolpopexia Suprapúbica en el manejo de la Incontinencia Urinaria de Esfuerzo de la Mujer, en la Clínica de Incontinencia del Servicio de Ginecología del Instituto Materno Infantil, de la Sección de Urología del Hospital San Juan de Dios y del Servicio de Ginecología de la Clínica Hospital Fray Bartolomé de las Casas, durante un período de 10 años, comprendidos entre el 1 de enero de 1978 al 31 de diciembre de 1987.

En la consulta Externa de Ginecología a las pacientes con diagnóstico clínico de incontinencia urinaria de esfuerzo se les practicó las pruebas de incontinencia y se les ordenó 
laboratorio de rutina, urocultivo, frotis vaginal y citología funcional para descartar y manejar patología asociada como diabetes, obesidad, infección urinaria, flujos genitales, hipoestrogenismo en las postmenopáusicas y luego son remitidas a la consulta de la Clínica de la Incontinencia donde se elabora Historia Clínica precodificada, examen físico y ginecológico, se hacen las pruebas de incontinencia de pie y de cúbito con $300 \mathrm{cc}$ de orina, se clasifica los grados de incontinencia y el tipo de incontinencia; se les realiza citoscopia a todas las pacientes, urografía a las que se les sospeche anormalidad del tracto urinario y a las que tienen infecciones urinarias a repetición, y Urodinamia en los casos en los cuales existen manifestaciones clínicas en donde es necesario descartar patología funcional (como urgencia, incontinencia de urgencia, enuresis, cirugía previa para incontinencia, incontinencia urinaria de esfuerzo después de histerectomía).

Se seleccionaron las pacientes con incontinencia urinaria de esfuerzo grado II y III para manejo quirúrgico a quienes se les ordenó ejercicios de Kegel preoperatorios, a las que se acompañaban de síntomas de inestabilidad vesical de tipo sensorial (con urodinamia normal) se les pidió valoración por neurología, reeducación vesical, y/o Bromuro de Propanteline por tres meses y luego se intervenían quirúrgicamente. Las pacientes cuya urodinamia mostró vejiga inestable recibieron tratamiento médico y se excluyeron del manejo quirúrgico.

Se observaron cuatro modalidades de procedimientos:

Teniendo de base la Uretrocolpopexia Suprapúbica (técnica Burch o Tanagho):

1. Colporrafia posterior.

2. Sin colporrafia posterior.

3. Con cirugía abdominal asociada.

4. Con histerectomía vaginal, operación de Manchester y corrección de celes.

En la evaluación de los resultados se tuvo en cuenta el grado del cirujano, el tipo de incisión, el material de sutura, el tiempo quirúrgico, la cirugía concomitante, las complicaciones intra y postoperatorias, la presencia de órdenes, la permanencia de la sonda, la estancia hospitalaria y se les practicó seguimiento a 1, 3, 6 meses, 1, 2, 5 y 10 años.

En cada control se evalúa la presencia de celes, el dolor de la cicatriz quirúrgica, la presencia de enterocele, y persistencia o recidiva de la incontinencia urinaria de esfuerzo tanto subjetiva como objetivamente con $300 \mathrm{cc}$ de orina se hacen las pruebas de incontinencia de pie y de cúbito.

\section{Resultados}

Información preoperatoria. Las pacientes a quienes se les practicó la uretrocolpopexia suprapúbica para la corrección de su incontinencia urinaria de esfuerzo tenían las siguientes características:

Edad. La menor de las pacientes tenía 27 años y la mayor 72 años. 229 (45.8\%) entre los 40-49 años y el promedio de edad fue de 46.62 años, (Figura No. 1).

Paridad. 481 (96.2\%) pacientes eran multíparas de las cuales el $75 \%$ tenían antecedentes de trauma obstétrico. Hubo $8(1.6 \%)$ casos de multíparas y $310(62 \%)$ de grandes multíparas. El promedio fue de 5.7 (Figura No. 2).

Evolución. El mínimo tiempo de evolución fue de 1 año y el máximo fue de 21 años. $348(69.5 \%)$ pacientes tenían una evolución menor de 5 años (Figura No. 3).

Figura No. 1

PACIENTES CON IUE* SE LES PRACTICO URETROCOLPOPEXIA SUPRAPUBICA EDAD

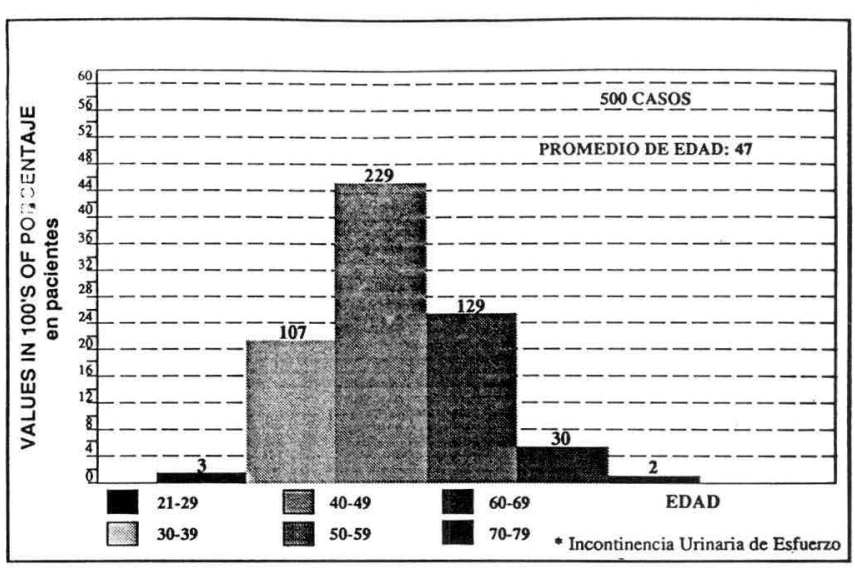

Figura No. 2

PACIENTES CON IUE* INTERVENIDAS QUIRURGICAMENTE PARIDAD
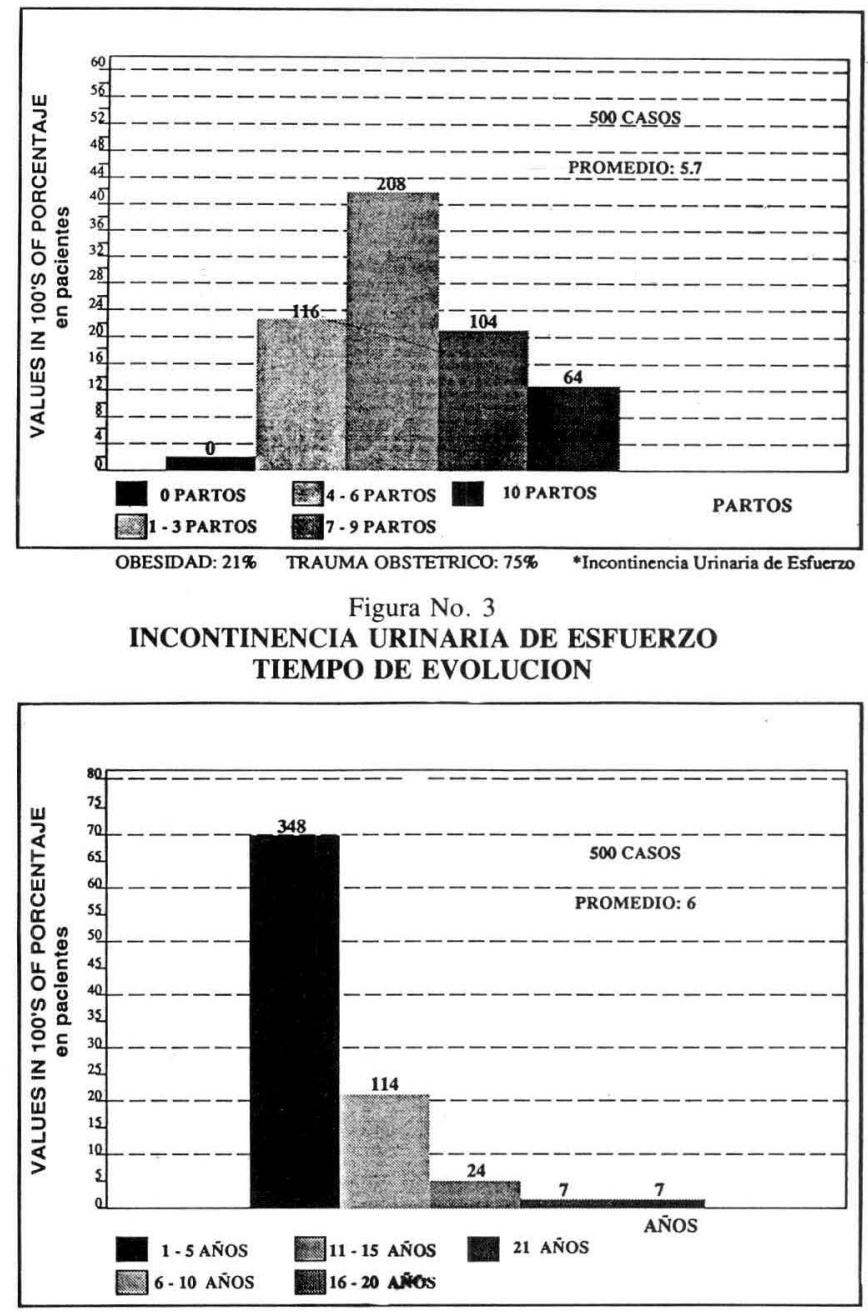
Obesidad. $105(21 \%)$ pacientes eran obesas.

Intervenciones previas. $39(7.8 \%)$ pacientes tenían antecedentes de operación de Kelly, 5 (1\%) Uretrocolpopexia suprapúbica, $2(0.4 \%)$ operación de Manchester y 16 (3.2\%). Histerectomía total abdominal (Tabla No. 1).

Tabla No. 1

INTERVENCIONES PREVIAS DE LAS PACIENTES CON IUE

\begin{tabular}{|lcr|}
\hline Tipo de investigación & No. de pacientes & $\%$ \\
\hline O. Kelly & 39 & 7.81 \\
Uretrocistopexia & 5 & 1.00 \\
Histerectomía & 16 & 3.2 \\
Manchester & 2 & 0.4 \\
\hline
\end{tabular}

\section{Diagnóstico clínico}

Tipo de incontinencia. $281(56.2 \%)$ pacientes tenían una incontinencia urinaria de esfuerzo (IVE) grado II y 219 $(43.8 \%)$ tenían IVE grado III (Figura No. 4 ).

Clasificación de los celes.

Uretrocistocele. $174(34.8 \%)$ pacientes tenían UCC grado I, $272(54.4 \%)$ grado II y $34(6.8 \%)$ grado III, (Figura No. 5).

Rectocele. $108(21.6 \%)$ no tenían celes, $213(42,6 \%)$ tenían rectocele y desgarro perineal grado I, $164(32,8 \%)$ grado II y 15 (3\%) grado III, (Figura No. 6). III.

Histerocele. $8(1.4 \%)$ pacientes tenían histerocele grado

Figura No. 4

INCONTINENCIA URINARIA DE ESFUERZO

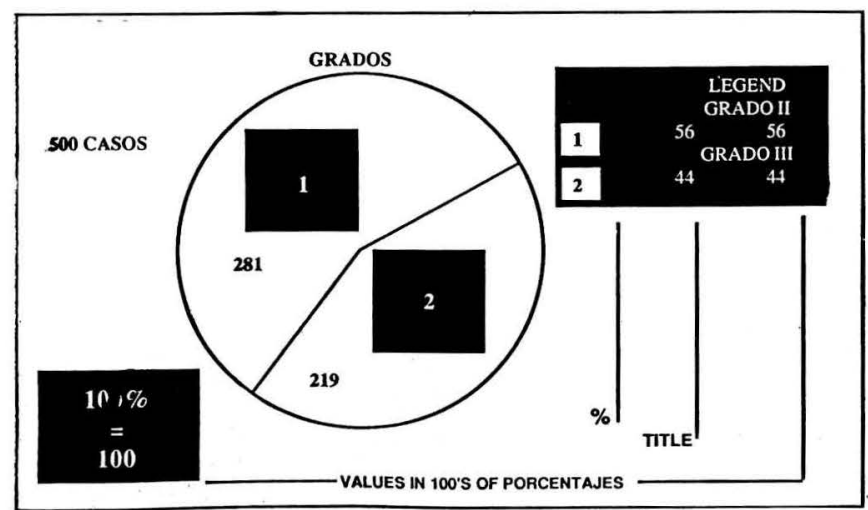

Figura No. 5

GRADO DE URETRO CISTOCELE 500 CASOS

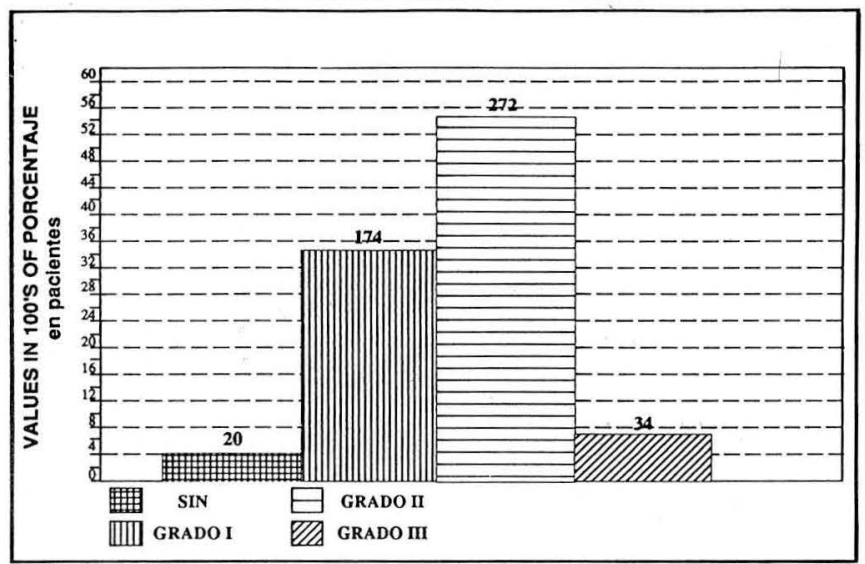

Figura No. 6

GRADO DE RECTOCELE 500 CASOS

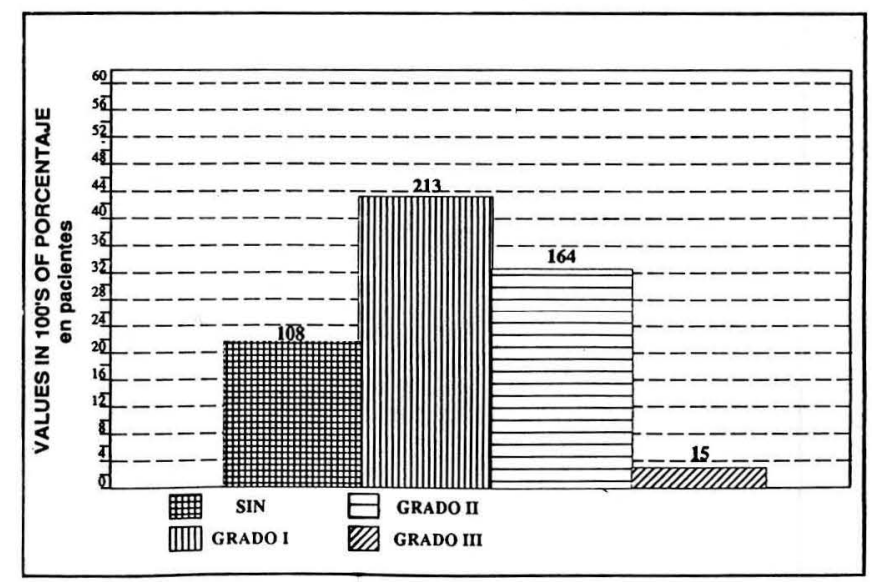

Otros diagnósticos. $46(9.2 \%)$ pacientes tenían además diagnóstico de miomatosis uterina, $5(0.1 \%)$ tenían tumor de ovario, $5(1 \%)$ tenían hiperplasia adenomatosa y $4(0.8 \%)$ Nic III.

Métodos paraclínicos preoperatorios. A la totalidad de las pacientes se les practicaron los exámenes de laboratorio preoperatorio habituales incluyendo la citología vaginal, frotis vaginal y el urocultivo, los cuales fueron negativos. A todas las pacientes se les practicó citoscopia descartando anormalidades uretrovesicales.

A $200(40 \%)$ pacientes se les practicó urografía excretora descartando anormalidades del tracto urinario alto.

A $56(11.2 \%)$ de las pacientes se les practicó urodinamia por existir sintomatología sugestiva de patología funcional.

Al comienzo del estudio se practicó cistometría a 98 (19.6\%) y se realizó a 70 (14\%) cistografía (Figura No. 7).

\section{Figura No. 7 \\ METODOS PARACLINICOS/DIAGNOSTICO \\ DE INCONTINENCIA URINARIA DE ESFUERZO EN LA MUJER 500 CASOS}

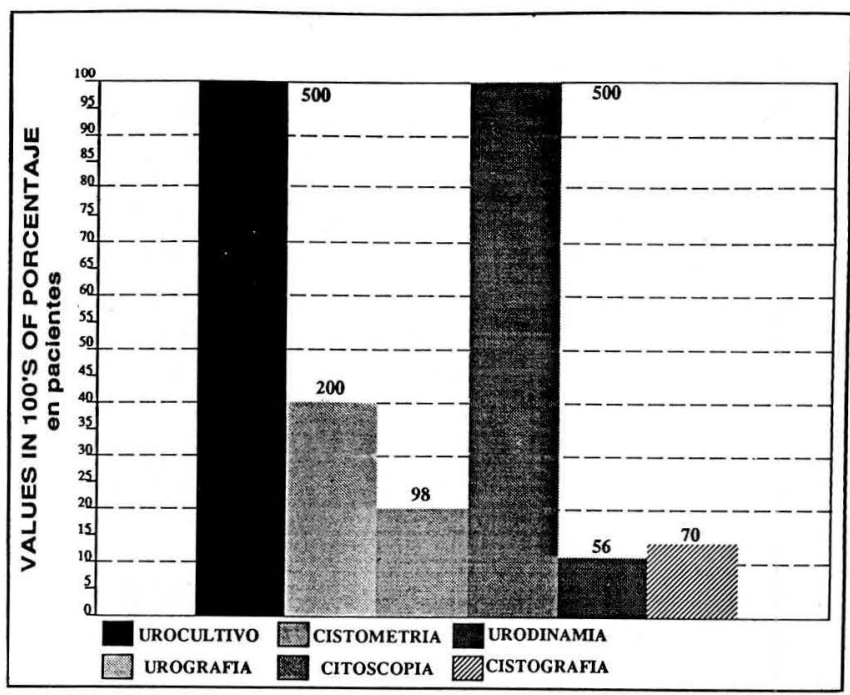




\section{Evaluación de la técnica quirúrgica (Uretrocolpopexia suprapúbica).}

Procedimiento quirúrgico. Se realizaron los siguientes procedimientos quirúrgicos de acuerdo con el diagnóstico concomitante (Figura No. 8).

1. De las 424 pacientes con incontinencia urinaria de esfuerzo acompañada de celes anterior y posterior I ó II, se realizó uretrocolpopexia suprapúbica únicamente en $178(35.6 \%)$ y además colpoperineorrafia posterior en $246(49.2 \%)$.

2. En las $7(1.47 \%)$ pacientes con prolapso genital III se realizó uretrocolpopexia suprapúbica más histerectomía vaginal más colpoperineorrafia posterior.

3. A $60(12 \%)$ pacientes se practicó histerectomía total abdominal por patología uterina y además colpoperineorrafia posterior.

4. Hubo 9 (1.8\%) casos a quienes se les practicó además Pomeroy.

Figura No. 8

PROCEDIMIENTOS QUIRURGICOS 500 CASOS

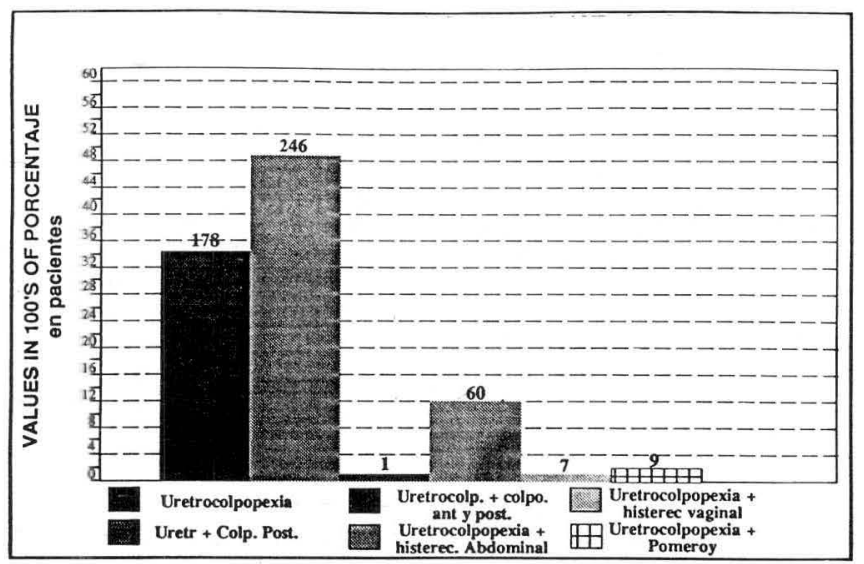

Tipo de incisión. A 227 (45\%) pacientes intervenida se les realizó incisión mediana y a $273(54.6 \%)$ se les practicó incisión de Phannestiels. (Tabla No. 2).

Tiempo quirúrgico. En caso de uretrocolpopexia suprapúbica el tiempo quirúrgico osciló entre 40 minutos y 120 minutos con un promedio de 89,4 horas. En caso de uretrocolpopexia más histerectomía abdominal osciló entre 1 y 4 horas con un promedio de 30 minutos para la colposuspensión. En caso de histerectomía vaginal osciló entre 90 minutos y 2 horas y media con un promedio de 1 hora y se utilizó dos equipos de cirujano (Tabla No. 2).

Cirujanos. Teniendo en cuenta que los procedimientos son hechos en hospital universitario, $259(51.8 \%)$ pacientes fueron intervenidas por Residentes tanto de Ginecología como de Urología y $241(48.2 \%)$ por Especialistas (Tabla No. 2)

Material de suturas. En 141 (28.2\%) pacientes se utilizó en sutura no absorbible y en 359 (71.8\%) no absorbible para la uretrocolposuspensión.

Tabla No. 2

EVALUACION DE LA TECNICA QUIRURGICA

Incisión:

Mediana

Pfannestiels

Material de suturas:

Absorbible

No absorbible

141

359

$28.2 \%$

$71.8 \%$

Tiempo quirúrgico de la uretrocolpopexia suprapúbica

\begin{tabular}{|c|c|c|}
\hline Sin colporrafia posterior & promedio & 85 minutos \\
\hline $\begin{array}{l}\text { Con colporrafia posterior } \\
\text { más histerectomía }\end{array}$ & promedio & 89 minutos \\
\hline $\begin{array}{l}\text { abdominal } \\
\text { más O. Manchester, } \\
\text { h. vaginal y/o }\end{array}$ & promedio & $2 \mathrm{H} 30$ minutos \\
\hline $\begin{array}{l}\text { colporrafia ant. y post. } \\
\text { más colporrafia } \\
\text { posterior }\end{array}$ & promedio & $1 \mathrm{H} 50$ minutos \\
\hline
\end{tabular}

\section{Cirujanos}

Residentes

Especialistas

259

$51.8 \%$

$241 \quad 48.2 \%$

Drenes

$\mathrm{Si}$

No

$478 \quad 95.6 \%$

$22 \quad 4.4 \%$

Medicamentos postoperatorios

$\mathrm{Si}$

No

$\begin{array}{ll}390 & 78.0 \% \\ 110 & 22.0 \%\end{array}$

Complicaciones intraoperatorias. Entre las complicaciones intraoperatorias se encontraron $8(1.6 \%)$ ruptura vesical las cuales fueron corregidas en el mismo acto quirúrgico, $26(5.2 \%)$ presentaron hemorragia importante y 42 $(8.4 \%)$ presentaron hematuria (Figura No. 9).

Figura No. 9

COMPLICACIONES INTRAOPERATORIAS 500 CASOS

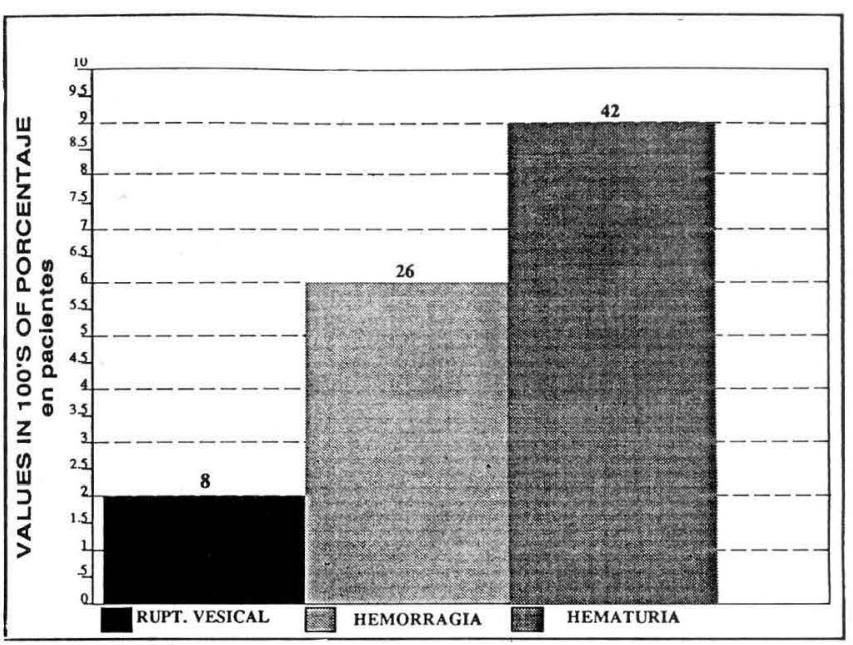


Medicación postoperatoria. Debido al uso postoperatorio de la sonda vesical, se utilizó antisépticos urinarios en 71 (14.2\%), en 295 (59\%) antibióticos y en 134 (26.85) no se utilizó medicación (Tabla No. 2)

Permanencia de la sonda vesical. El tiempo de permanencia de la sonda osciló entre 3 y 6 días en 420 , (84\%) (en caso de retención urinaria el tiempo osciló entre 7 y 16 días) en $24(4.8 \%)$ casos la permanencia fue de 48 horas y en el 56 (11.2) osciló entre 7 y 16 días en los casos graves de retención urinaria (Figura No. 10).

Figura No. 10

NUMERO DE DIAS DE PERMANENCIA DE SONDA VESICAL 500 CASOS

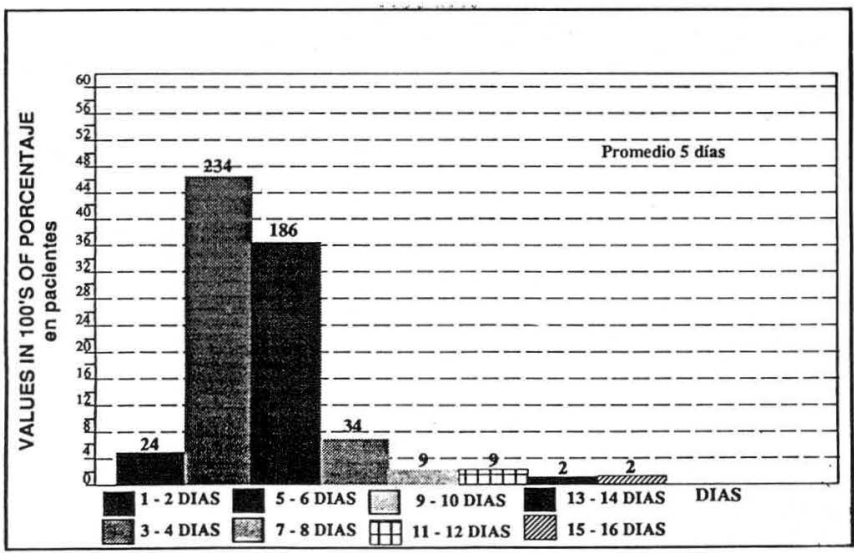

Complicaciones postoperatorias. 45 (9\%) pacientes presentaron absceso de paređ, 15 (3\%) presentaron infección de las colporrafias, $23(4.6 \%)$ presentaron hematomas de pared, hubo $84(16.8 \%)$ de retención urinaria las cuales fueron manejadas con sonda vesical y se les descartó infección urinaria 79 (15.8) presentaron infección urinaria 1 $(0.2 \%)$ presentó eventración (Figura No. 11).

Figura No. 11

COMPLICACIONES POSTOPERATORIAS DE LA URETROCOLPOPEXIA SUPRAPUBICA PACIENTES

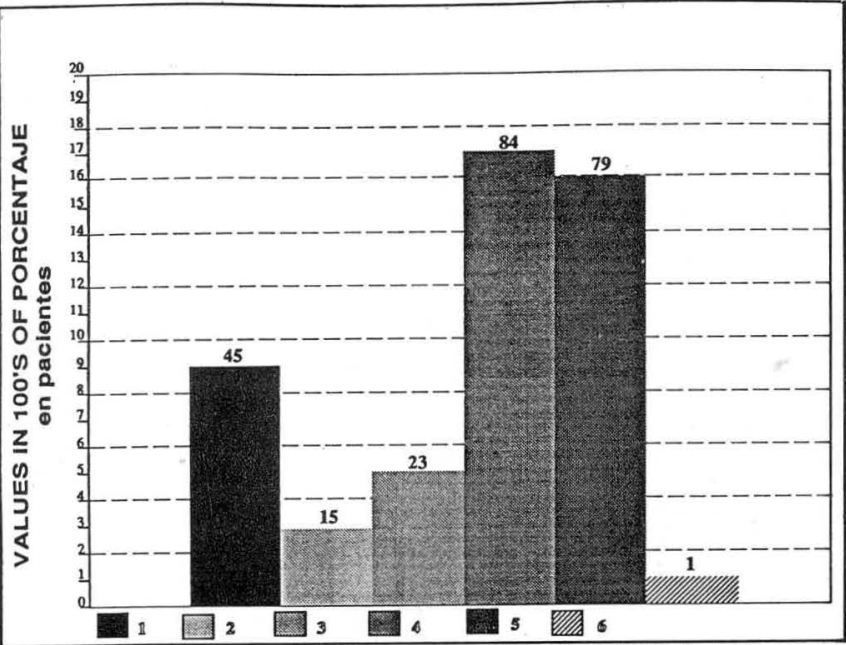

1 Infección Herida 2 Infección Colporrafias 3 Hematomas 4 Retención urinaria 5 Infección urinaria 6 Eventración
Infección urinaria. $79(15.87 \%)$ presentaron infección urinaria de las cuales $15(19 \%)$ tenían urocultivo. El germen más frecuente fue el E. Coli en 14 casos y en 1 caso fue Klebsiella (Figura No. 12).

El resto de pacientes tenían parcial de orina compatible con infección urinaria.

Retención urinaria. $73(14.6 \%)$ pacientes presentaron retención urinaria después del retiro de la sonda a los cuatro días de permanencia y el de las cuales 15 (20.54\%) tenían infección urinaria, las retenciones urinarias se manejaron con sonda vesical a permanencia y el tiempo osciló entre 1 y 10 días (Figura No. 11).

En ningún caso hubo necesidad de hacer dilataciones uretrales ni uretrotomía.

\section{Figura No. 12 \\ GERMENES INFECCION URINARIA DIAGNOSTICO DE LA INFECCION URINARIA POR UROCULTIVO}

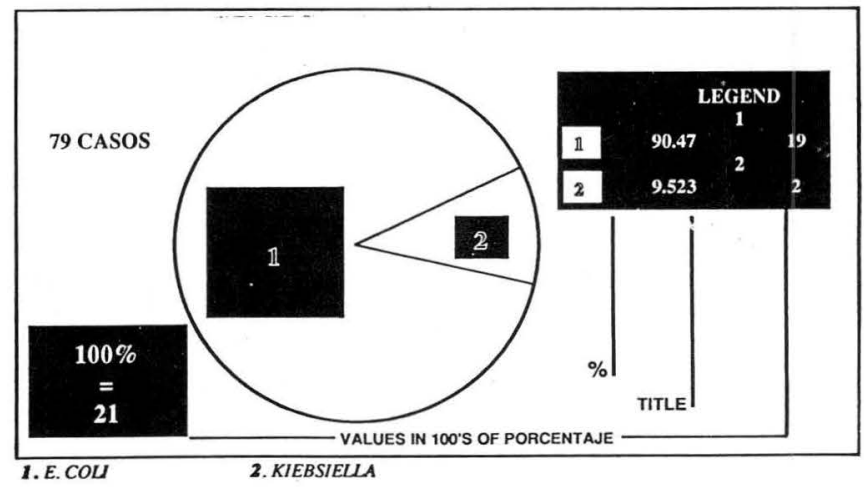

Residuio vesical $328(65.6 \%)$ tenían menos de $20 \mathrm{cc}$ de residuo vesical. 29 pacientes (5.8\%) tenían mayor de $60 \mathrm{cc}$ de residuo vesical (Figura No. 13).

Figura No. 13

RESIDUO VESICAL

POST-OPERATORIO

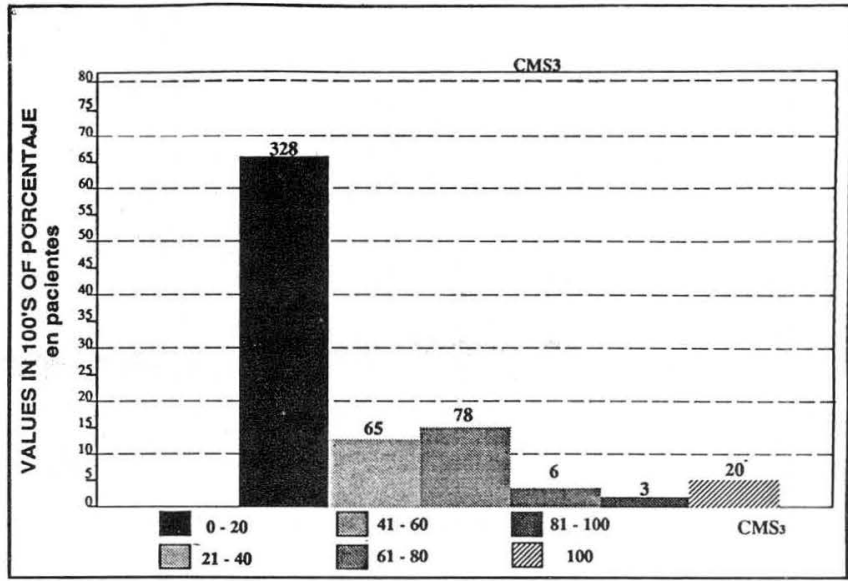

Estancia hospitalaria. En $127(25.4 \%)$ el promedio de estancia fue de 4 días, 234 (46.8\%) de 6 días, 61 (12.2\%) entre 7 y 16 días y $1(0.2 \%) 2$ días de estancia (Figura No. 14). 
Figura No. 14

ESTANCIA HOSPITALARIA

DE PACIENTES

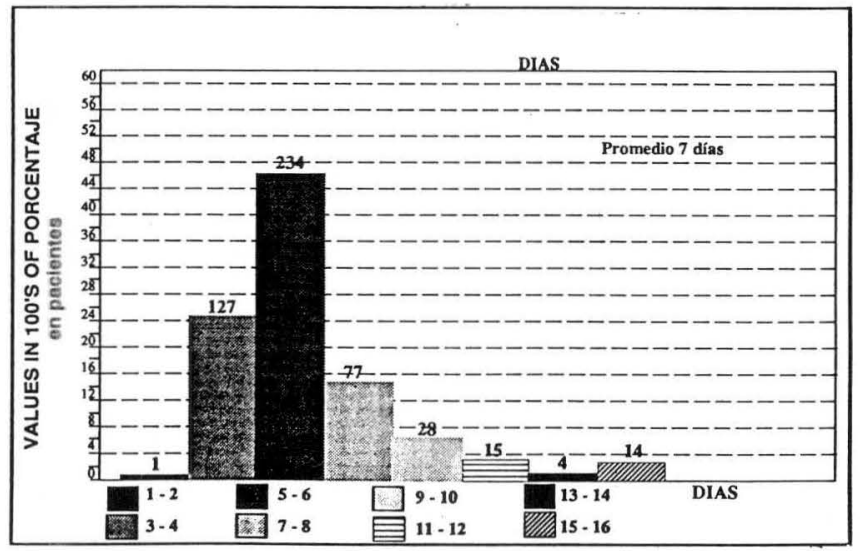

Seguimiento. $155(32.8 \%)$ pacientes tienen un seguimiento a un año, $124(24.85 \%)$ a 2 años, $154(31 \%)$ entre 3 y 5 años y $67(13.4 \%)$ entre 6 y 10 años. Lo cual se traduce que $325(67.2 \%)$ tienen un seguimiento a 2 años, $201(44.4 \%)$ a 3 años, y 67 (13.4\%) 5 años como mínimo (Figura No. 15).

Figura No. 15

SEGUIMIENTO DE LAS PACIENTES A QUIENES SE LES PRACTICO URETROCOLPOPEXIA SUPRAPUBICA

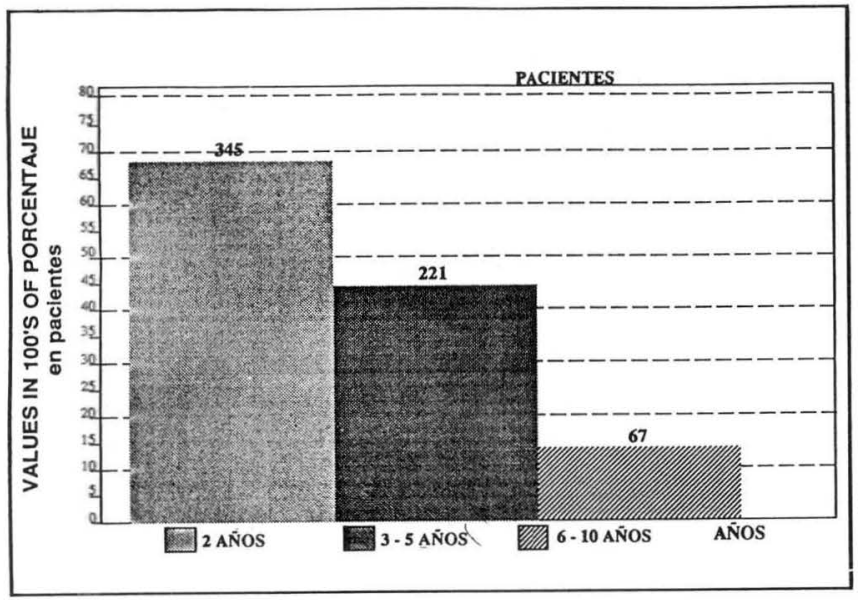

Eficacia. Las pacientes durante sus controles son valoradas subjetiva y objetivamente para descartar persistencia o recidiva de la incontinencia urinaria de esfuerzo: Se consideran curadas $482(96.4 \%)$ y hubo $18(3.61 \%)$ de fracasos (Figura No. 16).

Evaluación de los fracasos de la técnica quirúrgica. Se presentaron $18(3.4 \%)$ de fracasos con la técnica de la uretrocolpopexia suprapúbica cuyas características de estas pacientes fueron:

El $72.2 \%$ estaban entre los 40 y 49 años, el $33.3 \%$ tenía IVE grado II y el $66.6 \%$ grado III, se acompañaban de celes grado I en el $55.5 \%$ y UCC grado II en el $44.5 \%$, y el $22 \%$ tenían antecedentes de celes, y el $61.1 \%$ tenían síntomas de inestabilidad vesical.

Al $86.8 \%$ se les practicó Uretrocolpopexia suprapúbica únicamente y el $13.2 \%$ se les agregó la colporrafia posterior, el $77.5 \%$ las realizó el grupo Residente docente, el $77.7 \%$ se utilizó material no absorbible.

Dos pacientes tenían urodinamia normal en el preoperatorio y presentaron vejigas inestables en el postoperatorio comprobadas con urodinamia.

Se observó que el $83.3 \%$ se presentó antes de los seis meses y el $61 \%$ tenía además síntomas de inestabilidad vesical, el $5.5 \%$ al año y el $11 \%$ a los 6 y 7 años; éstas tres últimas con sintomatología de IVE pura.

Figura No. 16

EFICACIA Y FRACASOS

EN LAS PACIENTES

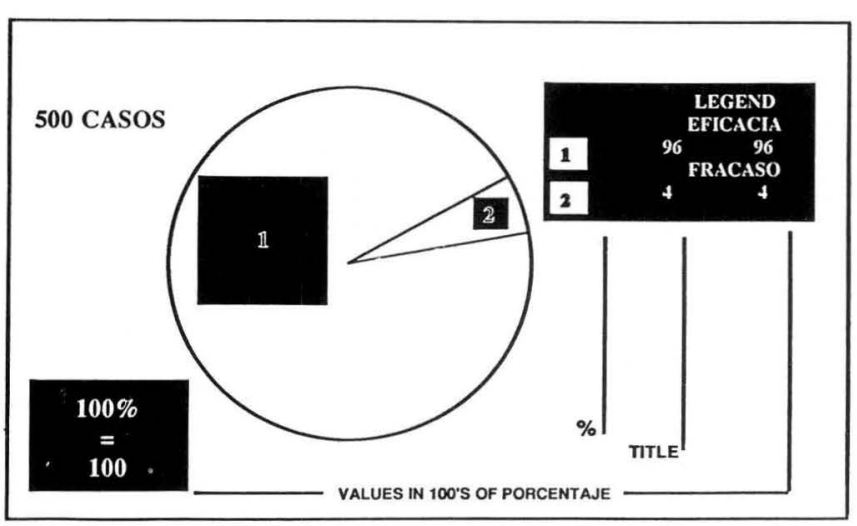

\section{Discusión}

1. La selección adecuada de las pacientes con IUE mediante una historia clínica precodificada, examen físico, neurológico completo y con ayuda de la citoscopia y urdinamia permitió una eficacia del $96.4 \%$ global en el manejo quirúrgico con la uretrocolpopexia suprapúbica más colporrafia posterior.

2. La Perimenopausia, íntimamente relacionada con la multiparidad y el tema obstétrico influyen en la aparición de la IUE, lo cual corrobora los datos de la literatura (21)

3. Durante el interrogatorio de las pacientes se debe investigar exhaustivamente los síntomas de inestabilidad vesical, los cuales son de mal pronóstico en el manejo quirúrgico de la IUE.

4. La uretrocolpopexia suprapúbica más colporrafia posterior es la técnica de elección en el manejo de la IUE de la mujer cuando se acompaña de los siguientes casos:

a. Relajación anatómica mínima de la uretra y de la unión uretrovesical.

b. En presencia de uretrocistocele I y II.

c. Cuando se acompaña de una indicación de cirugía abdominal como miomatosis uterina, tumor de ovario y cirugía general abdominal benigna.

d. Debilitamiento del sosten aponeurótico o ligamentoso de la uretra solamente pero sin pérdida del sostén anatómico demostrable del piso pélvico, cuando la paciente es nulípara, y generalmente postmenopáusica. 
e. Traumatismo quirúrgico de los ligamentos suspensorios procedimientos como vulvectomía radical.

f. Antecedente de colpopexia sacra para suspender una cúpula prolapsada con probable enderezamiento postoperatorio de la unión uretrovesical.

5. Se debe preferir una incisión de Pfannestiels en los casos que no se acompañe de cirugía abdominal, utilizar material, no absorbible, hacer hemostasia de las venas acompañarse siempre de una colporrafia posterior más miorrafia de los elevadores.

6. El tiempo promedio de cirugía fue de 85 minutos que está de acuerdo con los reportes de la literatura (39) pero es mayor que en los casos de la cervicosuspensión endoscópica (Stamey) y de la uretrocolpopexia intrapúbica (Powel).

7. En los casos de operación de Manchester, histerectomía vaginal y UCC grado III se necesitaron dos equipos quirúrgicos y agregó una hora más al procedimiento; la eficacia fue del $100 \%$ a dos años de los casos controlados, pero en estos casos se puede preferir técnica como la cervicosuspensión endoscópica o la uretrocolpopexia infrapúbica que mantienen la eficacia y disminuyen el tiempo quirúrgico y un equipo de cirujanos.
8. La uretrocolpopexia suprapúbica tiene mayor morbilidad que las técnicas vaginales $(25,40$ y 41$)$ y que la cervisuspensión endoscópica (34 y 42) pero su alta eficacia del $96.4 \%$ global y del $95 \%$ a dos años permite ser la primera elección en el manejo de la IVE en la mujer.

9. El $1.8 \%$ presentó perforación vesical intraoperatoria la cual se corrigió en el mismo acto quirúrgico y ninguna ha presentado fístula.

10. Las complicaciones postoperatorias inmediatas más importantes fueron la infección urinaria $15 \%$ y retención urinaria $17 \%$ y tardías el enterocele en el $44.4 \%$.

11. El manejo de la sonda vesical disminuyó de 6 a 4 días en la segunda mitad del estudio, sin aumentar la retención urinaria e influyendo en la disminución de la estancia hospitalaria de 6,5 a 4 días y de los costos hospitalarios.

12. El seguimiento de las pacientes a dos años aumentó del $38 \%$ al $67 \%$ lo cual nos ayuda a valorar la verdadera eficacia de la uretrocolpopexia suprapúbica.

\section{BIBLIOGRAFIA}

1. Te Linde. Incontinencia urinaria de esfuerzo, Uretrocele, Cistocele, Ginecología operatoria 1987; 25: 519-542.

2. Staskin DR., Simmern P., Hadley H., Raz S. Fisiopatología de la incontinencia urinaria de esfuerzo. Ginecología y Obstetricia, Temas actuales, $1985 ; 2:$ 385-396.

3. Bradley WE., Hald T. Urinary Incontinence. The Urinary Bladder: Neurology and Dynamics. 1982; 16: 175-203.

4. Smith P., Ch M. Age Changes in the female urethra. British Journal of Urology 1972; 44: 667-670.

5. McCarthy TA. Medical History and Physical examination. Gynecologic Urology and Urodynamics. Ostergard D. 1982; 4: 45-48.

6. Blaivas J. Urodynamic Testing. Female Urology, Raz Shlomo. 1983; 6: 79-99.

7. Walters $M$. and Shields $L$. The diagnostic value of history physical examination and the Q-tip cotton swab test in women with urinary incontinence. Am. J. Obstet. Gynecol. 1988; 159: 145-149.

8. Marchant DJ. Urinary Incontinence. In Pitkin RM, Zlatnik SJ. eds. Yearbook of obstetrics and gynecology. Chicago. 1981; 213.

9. Green TH. Development of a plan for the diagnosis and treatment of urinary strees incontinence. Am. J. Obstet. Gynecol. 1982; 83: 634-640.

10. Drutz HP. Do Static Cystourethrograms have a role in the investigation of female incontinence? Am. J. Obstet. Gynecol. 1978; 130 : 516-520.

11. Fantl JA. Bead chain Cystouretthrogram: an evaluation. Obstet. Gynecol. 1981; 58: 237-341.

12. Marchant DJ. Evaluación clínica de la Incontinencia Urinaria y de la anatomía y patofisiología anómalas. Clínicas Obstétricas y Ginecológicas. 1984; 2: 555-568.

13. McCarthy T. and Ostergard D. Office Urethroscopy. Gynecologic and obstetric urology. 1982; 6: 100-108.

14. Peter KS., Hill RC. and Ostergard DR. Supine Urethroscopic and Standing Cystometry as Screening Methods for the detection of Detrusor Instability. Obstet. Gynecol. 1987; 70: 57-60.

15. Robertson JR. Dynamic Urethroscopi. Gynecologic Urology and Urodinamics. Oster grad D.R. 1982; 11: 135-154.

16. Massey A. y Abrams P. Urodinámica de vías urinarias bajas. Ginecología y Obstetricia. Temas Actuales. 2: 347-369.
17. Obstergard DR. and McCathy TA. Diagnostic procedures in female urology. Am. J. Obstet. Gynecol. 1980; 137: 401-411.

18. Bhatiaen N. and Ostergard DR. Urodynamics in Women with Stress Urinary Incontinence. Obstet. Gynecol. 1982; 60: 552-559.

19. Wein AJ. Office Urodynamics. Urologic Clinics of North America Vol. 15 1988; (4): 609-623.

20. Hodgkinson CP. Stress Urinary incontinence. Am. J. Obstetrics and Gynecology 1970; 79: 648-664.

21. Harrison NW. Incontinencia de esfuerzo: pasado y presente. Ginecología y Obstetricia. Temas actuales 1978; 1: 65-103.

22. Stanton SL. Incontinencia de esfuerzo: por qué y cómo funcionan las operaciones. Ginecología y Obstetricia. Temas actuales. 1985; 2: 397-405.

23. Beck P. The sling operation. Gynecologic and obstetric Urology. 1982; 19: 285-308.

24. Stanton SL. and Cardozo L. A comparison of vaginal and suprapubic surgery in the correction of incontinence due to urethral sphincter incompetence. British Journal of Urology. 51: 497-499.

25. Kranst K. Operación de Marschall Marchetti Kranst. Complicaciones quirúrgicas en Obstetricia y Ginecología. Schaefer G. and Graber E. 1986; 27: 361-368.

26. Burch JC. Urethro vaginal fixation to Cooper \$ ligament for correction of incontinence, cystocele and prolapse. Am. J. of obstetrics and Gynecology. 1961; 81: 281-290.

27. Burch JC. Cooper \$ Ligament urethrovesical suspension for stress incontinence. Am. J. of obstetrics and Gynecology. 1968; 100: 764722.

28. Beck RP. and McCormick RN. Treatment of urinary stress incontinence with anterior colporraphy. Obstetrics and gynecology. 1982; 59: $269-274$

29. Gómez P y Lomanto A. Operación de Kelly. Revista Colombiana de Obstetricia y Ginecología. Vol. XXIV, 1983; 28.

30. Marshall VF., Marchetti AA., Krantz KE. The correction of stress incontinence by simple vesicourethral suspension. Surgery Ginecology and obstetrics.

31. Pereyra A. and Lebherz T. Combined Urethrovesical Suspension and Vaginourethroplasty for Correction of Urinary Stress Incontinence. Obst. Gynecol. 1967; 30(4): 537-545. 
32. Tanagho EA. and Cols. Colpocistouretropexia: La vía, Como la hacemos. J. Urol 1976; 116: 751-753.

33. Stamey TA. Endoscopic Suspension od the Vesical Neck for Urinary Incontinence. Surgery, Ginecology Obstetrics. 1973; 136: 547-554.

34. Stamey TA. Endoscopic Suspension of the vesical neck for urinary incontinence in females. Report on 203 consecutive patients. Annals of surgery. 1980; 192(4): 465-471.

35. Galloway NTM., Davies N., Stephenson TP. The Complications of Colposuspensión. British Journal of Urology. 1987; 60: 122-124.

36. Mundy AR. A trial comparing the stamey bladder neck suspension procedure with colposuspension for the treatment of stress incontinence. Br. J. Urol. 1983; 55: 687.

37. Weil A., Reyes H., Bischoff P. et al. Modification of urethral rest an stress profile after different types of surgery for urinary stress incontinence. Br. J. Obstet. Gynecol 1984; 91: 46.
38. Lomanto Morán A., Gómez CP. Evaluación de la Colpocistouretropexia en la incontinencia urinaria de esfuerzo. Revista Colombiana de Obstetricia y Ginecología. XXXII 1982; (2): 112-124.

39. Loughlin KR. and col. The Comparative Medical costs 2 major procedures available for the treatment of stress urinary incontinence. The Journal of Urology. 1982; 137: 436-438.

40. Stanton SL. Cirugía en la incontinencia urinaria. Temas actuales. Ginecología Obstetricia 1978; 1: 79-104.

41. Redondo $\mathrm{CH}$. Enfoque clínico y Terapéutico de la Incontinencia Urinaria de Esfuerzo en la Mujer. Urología Colombiana. 1987; 1: 29-47.

42. Lomanto MA. y Sánchez J. Evaluación de la Cervicosuspensión endoscópica. Trabajo no publicado 1988. 